\title{
Evaluating the Risk of Contracting Salmonellosis from Egg Yolk 'Parmesan' Based on Water Activity
}

Dianna Vuu ${ }^{1}$, Helen Heacock ${ }^{2}$

1 Lead Author, B. Tech Student, School of Health Sciences, British Columbia Institute of Technology

2 Supervisor, School of Health Sciences, British Columbia Institute of Technology

\section{Abstract}

Background: Egg yolk parmesan recipes have been gaining popularity since 2015. Most recipes include a heat treatment step which would kill egg-associated pathogens such as salmonella, however a significant number of recipes do not; resulting in a higher risk of salmonella growth and thus higher potential to cause food borne illness.

Methods: Salt-curing affects an intrinsic factor called water activity (Aw). At 0.93 Aw or below salmonella is unable to grow. This study measured the minimum amount of time required for the salt curing process to inhibit the growth of salmonella. To achieve this batches of egg yolk parmesan were made using varying curing durations and then the water activity of the finished product was measured. A one sample t-test statistical analysis was conducted to determine if, with $99 \%$ confidence, the water activity of yolks cured for the chosen duration can reliably reduce water activity below 0.93 .

Results: The minimum amount of time required for the water activity to decrease below 0.93 was 24 hours. Results were as follows: $\mathrm{N}=39$; the p-value is 0.0000000 and the power is 1.0000000 .

Conclusion: This is strong evidence to suggest that large grade A chicken egg yolks cured in a 74\% kosher salt and 26\% white granulated sugar mixture for 24 hours at refrigeration temperature will have a water activity below 0.93 . Therefore, it can be concluded that curing for 24 hours will inhibit potential salmonella growth.

Keywords: food safety, salmonella, salmonellosis, water activity, $\mathrm{pH}$, egg yolk parmesan, cured egg yolk, salt cured egg yolk.

\section{Introduction}

In modern times, food preservation techniques are no longer simply a means of extending shelf life by inhibiting pathogens and spoilage, but also a method of transforming ingredients to enhance their flavour. Egg yolk parmesan is a prime example of this. Its basic method of production involves resting a raw chicken egg yolk onto a thick layer of salt and/or sugar until the egg yolk has dehydrated into a firm solid puck (Birnbaum, 2018). This transformed ingredient is then sliced or grated onto finished pasta, salad, or ice cream as a garnish (Birnbaum, 2018; Food Insider, 2018; Kostow, 2015).

Dependent on the recipe, egg yolk parmesan may not undergo a heat treatment step (Food Insider, 2018; Kostow, 2015). In this case, the recipe relies solely on the ability of the desiccant to reduce the water activity to levels which are 
safe from pathogens. However, it is widely

known that chicken eggs are prone to

contamination by bacteria of the Salmonella

enterica spp. (Howard et al., 2012), a major

cause of foodborne enteric illness (BCCDC,

2016). Can this method of processing egg yolks

reliably inhibit the survival or growth of

Salmonella associated with chicken eggs?

\section{Literature Review}

Antimicrobial Properties of Chicken Eggs

The primary function of an egg is to house and support the growth of an embryo. The composition of a chicken egg can be divided into 3 main components: the shell and membranes, the albumin (aka. egg white), and the egg yolk (D. Chen, 2019; Wu, 2014). The shell, outer membrane, inner membrane, and vitelline membrane act as physical barriers. They help prevent the entry of contaminants into the egg and the loss of moisture out of the egg, while permitting transmission of oxygen (Howard et al., 2012; Wu, 2014). The vitelline membrane serves a secondary purpose to separate the yolk from the albumin; without the vitelline membrane, egg yolk would be liquid with no shape. Albumin provides a chemical barrier to inhibit microbial growth. It has a high $\mathrm{pH}$ of 8.4 to 9.3 and an inhibitor enzyme called lysozyme, both of which can help prevent the growth of pathogens like Salmonella (D. Chen, 2019). Finally, the yolk is the nutrient core of the egg. It is composed of approximately 50 to $52 \%$ water, $31 \%$ lipids, $16 \%$ proteins, carbohydrates and minerals (Anton, 2007), however, the exact composition depends on factors such as the hen's feed, breed, age, and the egg's size (Ahn et al., 1997; Lemahieu et al., 2015). This abundant source of nutrients creates an appealing environment for pathogens.

Egg yolks alone have no significant antimicrobial properties. There are no physical barriers inside the egg yolk, nor are there chemical inhibitory factors like in the albumin. The average $\mathrm{pH}$ of an egg yolk allows the growth of Salmonella (D. Chen, 2019), there are no significant inhibitor enzymes, and the high moisture and nutritional content allows microorganisms to grow and thrive. Thus, the vitelline membrane is the last line of defense within the egg to prevent pathogen growth. The vitelline membrane is affected by temperature (J. Chen et al., 2005). When eggs are stored at 4 degrees Celsius, the vitelline membrane requires more force for rupture, than when they are stored at 22 degrees Celsius. Vice versa, it is weakest when stored for a long time over moderate room temperatures, thus a cold storage is safer than its opposite.

\section{Egg Preservation}

Besides proper temperature control, the impact of unwanted microbiological activity can be reduced by altering the characteristics of the product so that it is unfavourable for the pathogen or spoilage organism of concern. For instance, water activity is a measure from 0.00 aw to $1.00 \mathrm{aw}$ of the free available water that can be used by microorganisms for metabolic reactions, allowing them to grow and proliferate. 
A water activity above 0.95 aw can sustain most bacteria, yeast, and mould, meanwhile a water activity of 0.85 aw limits the growth of most bacterial pathogens (Keilbart, 2017). Every microorganism has a sensitive range at which it can grow.

Some egg products which make use of this principle include salted eggs, miso cured eggs, and egg yolk parmesan.

Salted eggs begin with soaking a raw whole egg in brine or covering the egg with a salted charcoal paste, and then cooking the eggs before consumption (McGee, 2004; Ooi, 2012). The salt in the brine or the paste draws out moisture and lowers the water activity of the egg. Any microbes that survive these unfavourable conditions are then killed during the cooking process.

Miso-cured eggs, or misozuke eggs, use only the egg yolk. This method involves coating the raw yolk with a marinade made solely or predominantly with miso, a fermented soybean paste. It is then served raw (N. H. Chen, 2019). The miso-curing process primarily functions by inoculating the egg yolk with non-pathogenic microorganisms that will out-compete any new growth and secondly functions by lowering the water activity of the yolk. Miso curing originates from Japan, where raw eggs are commonly consumed and whole in-shell pasteurized eggs are available (Itoh, 2014).

\section{Egg Yolk Parmesan Method}

Egg yolk parmesan, salt-cured egg yolks, or also known as candied egg yolks use similar mechanisms as the previously mentioned methods, however, a lot of variation exists between the publicly available recipes. Chicken, duck, or goose egg yolks (Shaw, 2014) are separated and then placed onto a desiccant bed of salt, salt and sugar, or salt, sugar, herbs and spices (Flores, 2018). They are then allowed to rest for 4 hours, for a soft spreadable product (Egg Farmers of Canada, 2019), to 3 weeks, for a hard grateable product (Food Insider, 2018), in the refrigerator. After that, the excess desiccant is brushed and rinsed off. Some recipes will specify dehydrating the egg yolks further in an oven turned to its lowest heat setting (about $200^{\circ}$ F) (America's Test Kitchen, 2018; Egg Farmers of Canada, 2019), meanwhile, other recipes will deem the eggs ready for consumption without any heat treatment (Food Insider, 2018; Shaw, 2014). In recipes with heating, pathogens are inhibited by lowering water activity, and thermal inactivation. In recipes without heating, only water activity appears to be affected.

However, one resource states that salt-cured egg yolks also promote the growth of lactic acid bacteria in a manner similar to sauerkraut, a sour fermented cabbage product (Adamant, 2019). Sauerkraut is made by coating cabbage in salt and allowing natural microbial activity to ferment the vegetable over the course of 20 or more days (Howe, 2014). Lactic acid bacteria out-completes new spoilage and pathogenic microorganisms and lowers the $\mathrm{pH}$ of the product (Battcock \& Azam-Ali, 1998; Keilbart, 2017). 


\section{Salmonella}

The Salmonella enterica spp., often referred to as "Salmonella", is composed of approximately 100 different serotypes which cause human diseases (Duffy, 2019). However, the disease of most concern in egg products is Salmonellosis.

Salmonellosis is a zoonotic disease that is often transferred from animals such as poultry to humans via ingestion of contaminated food, water, or fecal particles (BCCDC, 2016; Duffy, 2019). Children under the age of 5 are at higher risk for salmonellosis, due to their hand to mouth habits. An infectious dose as low as 1000 bacilli can trigger the onset of symptoms (Public Health Agency of Canada, 2010). These symptoms often range from mild stomach cramping, diarrhea, nausea, to severe fever, vomiting, and dehydration (BCCDC, 2016). These symptoms contribute to an estimated 3 million deaths annually worldwide (Chimalizeni et al., 2010).

$S$. enteritidis is the most common serotype responsible for food-borne salmonellosis in chicken eggs in British Columbia (BCCDC, 2017; Whiley \& Ross, 2015). It first infects the hen via contaminated food or water sources and then colonizes the intestinal tract as it moves through the hen's digestive system. Once established, it will further invade into the intestinal epithelial cells. As an immune response, the hen's macrophages will attempt to remove the infection by enclosing the bacteria. However, Salmonella can survive and grow inside the macrophage, leading to a systemic spread as the macrophage migrates to other organs such as the ovary and cloaca (Gantois et al., 2009; Wu, 2014). There are two pathways which eggs are commonly infected. The first route being surface contamination of the eggshell with infected faecal matter. $S$. enteritidis can penetrate through the eggshell, inner membrane, and outer membranes. If the bacteria are not inhibited by the albumin, it may penetrate the vitelline membrane and colonize in the egg yolk. The second route is direct contamination of the yolk, albumen, egg membranes, or eggshell depending on the site of infection in the hen's reproductive tract (Gantois et al., 2009). There is a risk of $S$. enteritidis being present anywhere inside the egg.

\section{Risk of Salmonella on Egg Yolk Parmesan}

A theoretical approach can be taken to evaluate the risk of Salmonella on Egg Yolk Parmesan. Previous studies have established limits for Salmonella growth in terms of minimum, optimum, and maximum, temperature, $\mathrm{pH}$, and water activity conditions (Food Standards Australia New Zealand, 2013a). Adjusting the qualities of a food product to exceed these ranges (below the minimum, or above the maximum) will prevent Salmonella growth. Thus, if the Egg Yolk Parmesan method adequately exceeds these limits, it should be safe from $S$. enteritidis.

However, not all parameters are equal. Implementing maximum temperature $\left(46.2^{\circ} \mathrm{C}\right)$ will kill existing Salmonella but implementing the minimum water activity ( $0.93 \mathrm{aw})$ will only 
prevent further growth (Food Standards

Australia New Zealand, 2013a). Therefore, if

Salmonella has already invaded the egg yolk, and it has multiplied to an infectious dose before the Egg Yolk Parmesan has reached a water activity below $0.93 \mathrm{aw}$, there is potential for consumers to contract Salmonellosis from an Egg Yolk Parmesan product despite the low water activity. Additionally, while the $\mathrm{pH}$ limit for Salmonella ranges from 3.8 - 9.5, there are many complexities regarding $\mathrm{pH}$ as a control mechanism. The effectiveness of $\mathrm{pH}$ is dependent on not only the measured value, but also the type of acid used, temperature, fat content, salt content, and water activity (Keerthirathne et al., 2016). Therefore, the overall safety of Egg Yolk Parmesan can only be guaranteed with a combination of limiting parameters.

\section{Purpose of Study}

The purpose of this study was to evaluate the safety of Egg Yolk Parmesan based on the method's ability to reliably reduce water activity to levels which limit the growth of Salmonella enterica spp. (0.93 aw). The results of this study can be used to create public guidelines and establish a minimum curing duration which will minimize the risk of food-borne illness associated with this product.

In addition, $\mathrm{pH}$ data was collected to examine the possibility of lactic acid bacteria microbial activity which has been suggested as an important factor to the yolk preservation (Adamant, 2019).

\section{Materials and Methods}

\section{Recipe Comparison}

To ensure that the results of the study are representative of real-life practices, a comparison of existing recipes for egg yolk parmesan was conducted to aid in developing the method procedure and choosing the materials. A total of 32 recipes were compared. Similarities which appeared in the highest proportion were assumed to equate to the highest likelihood of being used.

\section{Materials}

The materials for this study were chosen to reflect the most probable real-life practices based on the recipe comparison data and commercial availability. Large-sized, grade A, regular (non-nutritionally enhanced) chicken eggs were the best choice to represent the egg yolks used in most Egg Yolk Parmesan recipes. The curing medium used in this study consisted of $74.4 \%$ (by weight) kosher salt \& $25.6 \%$ (by weight) white granulated sugar, which reflected the ratio and ingredients that were common to most Egg Yolks Parmesan recipes.

\section{Methods}

This study was conducted as a two phase experiment. The first phase determined the minimum curing time required for the water activity of the egg yolks to decrease to 0.93 and introduced the preparation procedure that was used throughout the study. The second phase determined if the "minimum curing time" was reliable. Water activity was measured using a AQUALAB 3TE water activity meter. 
Additional data such as weight, measured using a generic postal scale, and $\mathrm{pH}$, measured using HANNA Instruments Professional Portable Yogurt $\mathrm{pH}$ Meter, were also recorded throughout the study to ensure consistency in egg yolk volumes and the presence of lactic acid bacteria.

\section{Phase 1.}

The minimum curing time was examined by taking measurements of the egg yolk samples every 24 hours to monitor how the water activity changes and at which point the water activity is below 0.93 .

First, two raw untreated egg yolks were measured for weight, water activity, and $\mathrm{pH}$ to establish a control data group. The rest of the egg yolks (10) were then prepared using the egg yolk parmesan method without heat treatment. With clean dry hands, the egg yolks were separated from the egg and placed onto a bed of curing medium in a medium sized tray on top of a scale. Their weight was recorded by measuring the whole arrangement and taring the weight between each sample. When all the egg yolks were placed down, more curing medium was added to completely cover the surface of the egg yolks. The tray was then allowed to cure in a closed refrigerated environment.

After every 24 hours, egg yolks were removed, two at a time, from the curing mixture, cleaned of curing medium, and then measured. The cleaning involved gently brushing off the mixture using clean fingers and then rinsing the egg yolk in a room temperature water bath $\left(20^{\circ} \mathrm{C} \pm 5\right)$ for approximately 5 seconds. Any non-intact egg yolks were discarded. The measurement involved weighing and recording the weight of each egg yolk, and then individually mashing the samples within a plastic bag until each sample was homogenous. The samples were then spread evenly into the water activity sample and measured as per the device manual's instructions. The remaining sample portion from the bag was measured for $\mathrm{pH}$. These measurements continued until all the samples were used on day 7 .

The pilot study, conducted in the exact manner above, indicated that additional data between 0 to 24 hours of curing would be beneficial to the study. Another set of egg yolks were prepared and measured at bi-hourly intervals. The gathered data was examined together with the data for 24 hour curing intervals.

For analysis, scatter charts were created to compare curing time and water activity, and curing time and $\mathrm{pH}$ data. The earliest curing time at which the water activity of both samples was below 0.93 was used as the curing time for all the egg yolks during phase 2 . The $\mathrm{pH}$ data was examined for a significant decrease that may be attributable to lactic acid bacteria fermentation.

\section{Phase 2.}

The preparation and measurement procedure for the egg yolks in this portion of the study was the same as in Phase 1, with the exception that 42 eggs were cured for the duration found ( 24 hours) in the analysis of Phase 1 data. The water activity of these samples was statistically 
analyzed using NCSS statistical software and a one sample, one-tailed T-test with a $99 \%$ confidence level. The null and alternative hypothesis were as follows:

- $\mathrm{Ho}=$ Mean Aw $\geq 0.930$ - The cured yolks will have a water activity greater than or equal to 0.93 after 24 hours of curing.

- $\mathrm{Ha}=$ Mean Aw $<0.930$ - The yolks will have a water activity less than 0.930 after 24 hours of curing.

\section{$\underline{\text { Results }}$}

\section{Phase 1}

\section{$\underline{\text { Minimum Curing Time }}$}

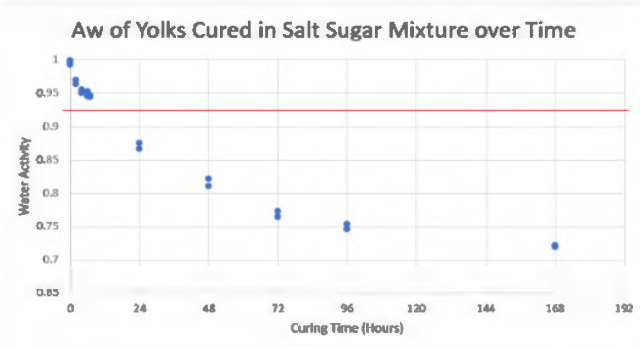

Figure 1. Phase 1 Aw over time to determine the curing time

The curing time and water activity, continuous numerical data, was plotted onto a scatter graph. An orange line was used to represent $0.93 \mathrm{Aw}$, the value at which Salmonella is unable to grow. It was found that 24 hours is the earliest curing time at which both data points are below 0.93 $\mathrm{Aw}$, thus this value was used as the curing time for all the egg yolks during Phase 2.

\section{Presence of Lactic Acid Bacteria}

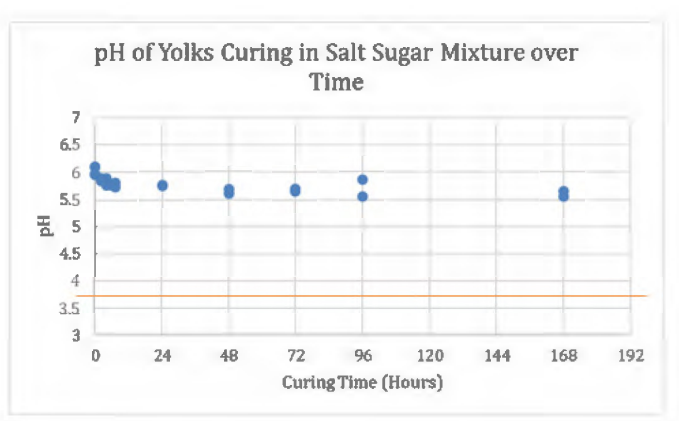

Figure 2. $\mathrm{pH}$ over Time to Determine the Presence of Microbial Activity

The curing time and $\mathrm{pH}$, continuous numerical data, was plotted onto a scatter graph. An orange line was used to represents $3.8 \mathrm{pH}$, the value at which Salmonella. is unable to grow. Studies on sauerkraut fermentation have shown that lactic acid bacteria will cause a rapid drop in $\mathrm{pH}$ within 20 hours and should fall below 4.5 within 60 hours (Jagannath et al., 2012; Wiander \& Korhonen, 2011). The $\mathrm{pH}$ data of the cured egg yolks have shown an insignificant decrease over the duration of 7 days in comparison to these values. Therefore, it is unlikely that microbial activity has occurred on the egg yolk samples that would protect them from the growth of

\section{Salmonella.}

\section{Phase 2}

Statistical analysis using NCSS revealed that the data was normally distributed. $99 \%$ of egg yolks, cured in the same manner as this study, will have a water activity between the confidence interval of $0.846-0.864$. The p-value was 0.00000 , thus the null hypothesis was rejected, and it can be concluded that the yolks will have a water activity less than 0.930 after 24 hours of 
curing. This result had a power of 1 meaning that this conclusion was very robust.

\section{Discussion}

This study provided strong evidence that 24 hours is the minimum curing time required to reduce the risk of Salmonella enterica spp. growth on an egg yolk. However, while this data is very robust, it is important to consider the applicability of this data to real life practices, the limitations in time and equipment resources, and any potential sources of error that exist in the study, before adopting these results into publicly distributed guidelines.

The study also showed some support against the notion of lactic acid bacterial activity, but this data was subject to several limitations which make it difficult to fully disprove the claim. Overall, there is still room for this topic area to be expanded and improved. Future studies can be conducted on aspects of Egg Yolk Parmesan recipes that have yet to be investigated.

\section{Applicability of Results}

The preparation methods used in this study make the data applicable to a wide range of Egg Yolk Parmesan recipes. Recipes are conducted either with or without heat treatment. They share common preparation steps up to the moment where the eggs are left to cure for a set duration of time. Based solely on the control mechanisms involved, recipes with heat treatment will create safer products for consumption than recipes without heat treatment. Since this study follows only the common steps, the results are more reflective of the riskier practice but correspond to both categories of procedures.

The specificity of the ingredients used in this study may pose limits on the relevancy of the data. The selection of egg type and curing medium ingredients can affect many outcomes, including the total amount of moisture, the rate of curing and water loss, and the flavor profile. Thus, an in-depth examination of each ingredient would be useful for the creation of new salt-cured egg yolk recipes. However, introducing multiple factors into the experiment will make interpretation of the results more complex. By experimenting with only one selection of ingredients, the focus of the study is placed on only one factor of the recipe and the results yielded are robust and simple.

Quality-wise, there tend to be two textures that people aim for when making egg yolk parmesan and both can be achieved even when using a minimum curing time of 24 hours. These textures are the soft spreadable egg yolk, which recipes may require as little as 4 hours to cure, and the hard grateable egg yolk which requires heat treatment or up to 3 weeks of curing. In this study it was found that after 4 hours of curing the water activity was not low enough to inhibit Salmonella growth, therefore more data within the 0 - 24 hour time frame would have been beneficial. However, after 24 hours of curing, the egg yolks were still soft enough to use as a spread and did have a low enough water activity to inhibit the pathogen, indicating that the desired soft texture is still achievable via a safer, 
timeframe. Hard grateable egg yolks require curing for longer than 24 hours or the addition of a further drying step like the use of an oven or dehydrator. This means that a minimum curing time of 24 hours is versatile and implementing this guideline is reasonable and necessary for recipes that produce soft spreadable yolk products.

\section{Presence of Lactic Acid Bacteria}

Lactic acid bacteria activity has been suggested as a factor to the effectivity of the Egg Yolk Parmesan method in the same manner which sauerkraut is fermented (Adamant, 2019). The gathered data shows that this notion is unlikely because sauerkraut fermentation results in a notable drop in $\mathrm{pH}$ within the first 60 hours, but no notable decline in $\mathrm{pH}$ occurred on the egg yolk samples after 7 days of curing. It is possible that temperature and time were factors that limited the potential growth of lactic acid bacteria. Sauerkraut fermentation is typically conducted at room temperature between $18-22^{\circ} \mathrm{C}$, the ideal temperatures for lactic acid bacteria, for a minimum of 20 days (Howe, 2014). In comparison, the longest time that the egg yolks were cured for was 7 days and all curing took place in a refrigerated environment. The author who made this claim of lactic acid bacteria used a recipe which involved curing egg yolks in a refrigerator for 7 days then leaving the egg yolks exposed to air at room temperature for another 7 to 10 days. It is possible that had the samples been cured for longer and/or cured in a room temperature environment, a reduction in $\mathrm{pH}$ may be observed. Thus, the claim of microbial activity cannot be entirely disproven.

\section{Limitations}

The limitations to gathering accurate data in this study falls upon time restrictions, scope restrictions and the potential for error inherent in manual sample preparation.

Time was a major limiting factor to data collection. Data between $8-23$ hours and days 5 and 6 could not be captured due to timing conflicts since the equipment was only accessible on the BCIT campus and during working hours. This missing data could only be collected outside of the available time. The scope of the study focused solely on Salmonella enteritidis, however Staphylococcus aureus is another pathogen of concern. It is able to survive in low water activity, high salt environments, and able to form heat stable toxins (Food Standards Australia New Zealand, 2013b). Thus, it is also relevant for egg yolk parmesan.

There are several steps in the sample preparation that rely on human activity and thus hold potential for inconsistency errors. The salt and sugar curing medium was manually wiped and rinsed off of the egg yolk samples. It was possible that some samples had absorbed excess water or were not entirely cleaned of salt in the process, which is why a maximum duration of 5 seconds in the water bath was implemented. It is also possible that during the manual homogenizing step, the samples were not mixed 
well enough to provide a representative data point of the complete egg yolk. To counteract this error, each individual sample was carefully inspected using visual and tactile qualities to minimize discrepancies. Both errors could have been eliminated with the use of automated equipment for these steps, however no such equipment was available or likely to exist. Therefore, human error may have occurred during the experiment, however precautions were made, where possible, to ensure accurate results.

\section{Knowledge Translation}

These results can be used to form a publicly accessible guideline on creating safer egg yolk parmesan. Health officials may use this guideline during food service establishment inspections, and professional chefs and members of the public may use this guideline when they create their own egg yolk parmesan. This document will allow them to more easily recognize and avoid risky practices. Meanwhile, the publication of this report can fill the current knowledge gap on the egg yolk parmesan preservation method. Overall, the distribution of this information should help to reduce potential cases of salmonellosis attributed to egg yolk parmesan.

\section{Recommendations for Future Studies}

The scientific knowledge base of egg yolk parmesan can be expanded upon by conducting further studies regarding the effects of:

- Changing the curing medium ratio and/or ingredients
- Changing the size, breed, or using nutritionally enhanced eggs

- Changing the volume of curing medium surrounding the egg yolk

- Curing the egg yolks at room temperature vs. refrigeration temperatures.

- Applying heat treatment

- The egg yolk parmesan preservation method on Staphylococcus aureus

\section{Conclusion}

In conclusion, implementing a curing time minimum of 24 hours for egg yolk parmesan will effectively reduce the risk of Salmonella enterica spp. by preventing any further growth, based on the water activity being less than 0.93 . This is applicable for a wide range of recipes, from those with heat treatment to those without, and for both soft and hard textured cured egg yolks. Health officials, chefs, and members of the public will benefit from the release of a food safety message to reduce the likelihood of food borne illness induced by egg yolk parmesan. The examination of natural lactic acid bacteria activity based on $\mathrm{pH}$ was inconclusive. While Salmonella can be controlled by the salt curing method, further studies on other pathogens or parameters are needed to deem egg yolk parmesan $100 \%$ safe for all people.

\section{Acknowledgement}

This project would not have been made possible without the generous support of many individuals. Thank you, Ken Keilbart, for providing recommendations and consultation throughout the project; Helen Heacock, for 
providing supervision and constructive feedback on the report; Fred Shaw, for providing lab supervision; and Melinda Lee and BCIT, for providing the necessary lab equipment.

\section{Competing Interest}

The authors declare that they have no competing interests.

\section{References}

Adamant, A. (2019, January 3). Salt Cured Egg Yolks. Practical Self Reliance.

https://practicalselfreliance.com/salt-cured-eggyolks/

Ahn, D. U., Kim, S. M., \& Shu, H. (1997).

Effect of egg size and strain and age of hens on the solids content of chicken eggs. Poultry

Science, 76(6), 914-919.

https://doi.org/10.1093/ps/76.6.914

America's Test Kitchen. (2018). Salt-Cured Egg

Yolks.

https://www.splendidtable.org/recipes/saltcured-egg-yolks

Anton, M. (2007). Composition and Structure of Hen Egg Yolk. In R. Huopalahti, R. LópezFandiño, M. Anton, \& R. Schade (Eds.), Bioactive Egg Compounds (pp. 1-6). Springer Berlin Heidelberg. https://doi.org/10.1007/9783-540-37885-3_1

Battcock, M., \& Azam-Ali, S. (1998).

Fermented and vegetables. A global perspective.

Chapter 5. Food and Agriculture Organization of the United Nations Rome.

http://www.fao.org/3/x0560e/x0560e10.htm

BCCDC. (2016, June 20). Salmonella Infection. http://www.bccdc.ca/health-info/diseasesconditions/salmonella-infection

BCCDC. (2017). Salmonella (non-typhoidal) Summary for 2017. http://www.bccdc.ca/healthprofessionals/data-reports/reportable-diseasesdata-dashboard?Disease $=$ Salmonella $+($ nontyphoidal)

Birnbaum, M. (2018, February 1). America's Test Kitchen simplifies salt-cured egg yolks [Interview]. https://www.splendidtable.org/story/americastest-kitchen-simplifies-salt-cured-egg-yolks

Chen, D. (2019, October). Eggs [In person].

Chen, J., Thesmar, H. S., \& Kerr, W. L. (2005). Outgrowth of Salmonellae and the Physical Property of Albumen and Vitelline Membrane as Influenced by Egg Storage Conditions. Journal of Food Protection, 68(12), 2553-2558. https://doi.org/10.4315/0362-028X-68.12.2553

Chen, N. H. (2019, February 28). Tsukemono Misozuke (Miso Pickling) 味噌漬け• Just One Cookbook. Just One Cookbook. https://www.justonecookbook.com/tsukemonomisozuke-miso-pickling/

Chimalizeni, Y., Kawaza, K., \& Molyneux, E. (2010). The Epidemiology and Management of 
Non Typhoidal Salmonella Infections. In A.

Finn, N. Curtis, \& A. J. Pollard (Eds.), Hot

Topics in Infection and Immunity in Children VI

(pp. 33-46). Springer New York.

https://doi.org/10.1007/978-1-4419-0981-7_3

Duffy, S. (2019, March). BHSC 7423 Unit 4

Introduction to Foodborne Illnesses [Lecture].

Egg Farmers of Canada. (2019). Salt Cured Egg

Yolks. Eggs.Ca.

https://www.eggs.ca/recipes/salt-cured-egg-

yolks

Flores, E. K. (2018, March 15). Cured Egg

Yolks. Adventures in Cooking.

https://adventuresincooking.com/cured-egg-

yolks/

Food Insider. (2018, April 22). Would You Try

Candied Egg Yolks? Food Insider.

https://www.youtube.com/watch?v=xABkcSr47

$\mathrm{dM}$

Food Standards Australia New Zealand. (2013a).

Salmonella (non-typhoidal).

https://www.foodstandards.gov.au/publications/

Documents/Salmonella\%20(non-typhoidal).pdf

Food Standards Australia New Zealand.

(2013b). Staphylococcus aureus.

https://www.foodstandards.gov.au/publications/

Documents/Staphylococcus\%20aureus.pdf

Gantois, I., Ducatelle, R., Pasmans, F.,

Haesebrouck, F., Gast, R., Humphrey, T. J., \&

Immerseel, F. van. (2009). Mechanisms of egg contamination by Salmonella Enteritidis. FEMS

Microbiology Reviews, 33(4), 718-738.

https://doi.org/10.1111/j.1574-

6976.2008.00161.x

Howard, Z. R., O’Bryan, C. A., Crandall, P. G., \& Ricke, S. C. (2012). Salmonella Enteritidis in shell eggs: Current issues and prospects for control. Food Research International, 45(2), 755-764.

https://doi.org/10.1016/j.foodres.2011.04.030

Howe, H. (2014, September 26). How Long To Ferment Sauerkraut? MakeSauerkraut.

https://www.makesauerkraut.com/how-long-toferment-sauerkraut/

Itoh, M. (2014, September 16). The raw appeal of eggs. The Japan Times Online.

https://www.japantimes.co.jp/life/2014/09/16/fo od/raw-appeal-eggs/

Jagannath, A., Raju, P. s., \& Bawa, A. s. (2012).

A two-step controlled lactic fermentation of

cabbage for improved chemical and

microbiological qualities*. Journal of Food

Quality, 35(1), 13-20.

https://doi.org/10.1111/j.1745-

4557.2011.00427.x

Keerthirathne, T. P., Ross, K., Fallowfield, H., \& Whiley, H. (2016). A Review of Temperature, $\mathrm{pH}$, and Other Factors that Influence the

Survival of Salmonella in Mayonnaise and Other Raw Egg Products. Pathogens, 5(4).

https://doi.org/10.3390/pathogens5040063 
Keilbart, K. (2017). Food Processing Lecture

[In person].

Kostow, C. (2015, November). Cured Egg Yolks

Recipe. Bon Appetit.

https://www.bonappetit.com/recipe/cured-egg-

yolks

Lemahieu, C., Bruneel, C., Ryckebosch, E.,

Muylaert, K., Buyse, J., \& Foubert, I. (2015).

Impact of different omega-3 polyunsaturated

fatty acid (n-3 PUFA) sources (flaxseed,

Isochrysis galbana, fish oil and DHA Gold) on

n-3 LC-PUFA enrichment (efficiency) in the egg

yolk. Journal of Functional Foods, 19, 821-827.

https://doi.org/10.1016/j.jff.2015.04.021

McGee, H. (2004). On Food and Cooking.

http://wtf.tw/ref/mcgee.pdf

Ooi, L. (2012, April 8). Salted Eggs. Roti n

Rice. https://www.rotinrice.com/salted-eggs/

Public Health Agency of Canada. (2010,

September 17). Pathogen Safety Data Sheets:

Infectious Substances - Salmonella enterica spp.

[Education and awareness;guidance]. Aem.

https://www.canada.ca/en/public-

health/services/laboratory-biosafety- biosecurity/pathogen-safety-data-sheets-riskassessment/salmonella-enterica.html

Shaw, H. (2014, May 9). Cured Egg Yolk

Recipe-How to Salt Cure Egg Yolks | Hank

Shaw. Hunter Angler Gardener Cook.

https://honest-food.net/salt-cured-egg-yolks/

Whiley, H., \& Ross, K. (2015). Salmonella and

Eggs: From Production to Plate. International

Journal of Environmental Research and Public

Health, 12(3), 2543-2556.

https://doi.org/10.3390/ijerph120302543

Wiander, B., \& Korhonen, H. (2011). A

Preliminary studies on using LAB strains

isolated from spontaneous sauerkraut

fermentation in combination with mineral salt, herbs and spices in sauerkraut and sauerkraut

juice fermentations. Agricultural and Food

Science, 20.

https://doi.org/10.2137/145960611797215682

$\mathrm{Wu}$, J. (2014). Eggs and Egg Processing. In Food Processing: Principles and Applications

(Second, pp. 437-455). John Wiley \& Sons, Ltd. https://pdfs.semanticscholar.org/dcf1/9d5ff3848 9a3fa7517b258df603c6004e6ab.pdf

Vuu, D. (2020). Evaluating the Risk of Contracting Salmonellosis from Egg Yolk 'Parmesan' Based on Water Activity. BCIT Environmental Health Journal. 